\title{
Estudio comparativo de la eficiencia de dos mediadores en la formación de docentes, utilizando un mismo ambiente de aprendizaje, en el diseño de competencias
}

\author{
Autor: PÉREZ SÁNCHEZ, Elkin Olaguer
}

Palabras claves: Diseño de competencias

\section{Descripción}

Informe final de investigación de tesis de maestría en donde se propone un estudio comparativo de la eficiencia de dos mediadores en la formación de docentes, utilizando un mismo ambiente de aprendizaje, en el diseño de competencias, software que fue desarrollado utilizando Flash.

A partir de la revisión del estado del arte y de la fundamentación en un marco conceptual, se identifican las dimensiones cualitativas y cuantitativas a investigar, se hace uso de la estadística y la observación no estructurada para obtener los resultados y se procede a hacer el análisis de datos. La parte final presenta las conclusiones y recomendaciones originados del trabajo investigativo.

\section{Contenidos}

El informe de investigación corresponde al esquema de trabajo de tesis. Se presenta una justificación y estudios relacionados con experiencias alrededor de formación de docentes utilizando aplicativos, aunque no se encontraron antecedentes directos de formación de docentes para diseñar competencias utilizando un aplicativo. En el marco de referencia se presentan un recorrido histórico por diferentes experiencias en la formación de docentes en el ámbito nacional e internacional, el concepto de competencias para la educación superior, los componen- tes normativos para el diseño de competencias, la fundamentación conceptual del diseño de competencias, el aprendizaje autórregulado, el diseño como dominio de conocimiento, los ambientes de aprendizaje y la eficiencia

\section{Metodología}

Teniendo en cuenta que el objetivo es comparar la eficiencia que tiene el tipo de mediador utilizado, por dos grupos de docentes, en el diseño de competencias, se utiliza una metodología de tipo cualitativo ya que se hace una valoración de todas las perspectivas dentro de la misma, se atiende un caso particular, se desarrolla en contextos naturales y es fundamentalmente interpretativa, prestando especial atención al contexto en el cual se desempeñan los participantes.

De acuerdo con las clasificaciones establecidas por diversos autores se puede plantea que es de corte cualitativo, ya que utiliza una metodología empírico - analítica, donde se trabajó con dos grupos en el mismo ambiente computacional y se hacen revisiones para cada grupo. Se consideran como variables independientes los tipos de mediadores utilizados por los docentes para el aprendizaje del diseño de competencias y como variable dependiente la eficiencia para el diseño de las competencias. 


\section{Conclusiones}

Entre otras se pueden destacar:

- No existen diferencias significativas en la eficiencia con la que dos grupos de docentes diseñaron las competencias, ya que tanto los que interactuaron con el software, bajo la orientación del profesor y los que interactúan con el software, de manera autorregulada lograron un nivel similar de aprendizaje en el diseño de competencias, de acuerdo con el experimento propuesto y la prueba t realizada.

- Los docentes del grupo sin tutor actuaron de manera autorregulada ya que guiaron su propia actuación a metas (aprender a diseñar competencias) a lo largo del tiempo que duro la experimentación.

- Es significativo que los docentes que trabajaron con el software de manera autorregulada generaron procesos de aprendizaje positivos muy cercanos a tiempos óptimos y resultados aceptables en niveles de apropiación de las competencias.

- El desarrollo de aplicativos y software educativos, hace posible atender intereses de aprendizaje de los individuos, para lograr una mayor permanencia de los conocimientos.

- Existen diferencias poco significativas en el diseño de competencias entre los docentes que utilizan un software con la dirección del tutor para el diseño de competencias y los que utilizan el mismo software pero de manera autorregulada

- Se requieren futuras investigaciones en donde se realicen estudios en profundidad con el fin de abordar desde otras perspectivas las dimensiones manejadas en la comprensión lectora en esta investigación. 